\title{
Frequency dependent Dielectric Properties of Ni-Zn-Co ferrite and Barium Titanate ferroelectric ME composites
}

\author{
N. M. Burange*, R. K. Pinjari, B. A. Aldar \\ Department of Physics, Smt. Kasturbai Walchand College, Sangli, Maharashtra, India.
}

\begin{abstract}
ME}$ Composites of $y\left(\mathrm{Ni}_{0.5} \mathrm{Zn}_{0.3} \mathrm{Co}_{0.2} \mathrm{Fe}_{2} \mathrm{O}_{4}\right)+(1-y)\left(\mathrm{BaTiO}_{3}\right)$ with $\mathrm{y}=0.1,0.2,0.3,0.4$ and 0.5 were prepared by standard double sintering method. The interfaces play very important roles in the dielectric properties, causing space charge effects and Maxwell-Wagner relaxation, particularly at low frequencies. The presence of ferrite and ferroelectric phases without any impurity was confirmed by X-ray diffraction. Because of the hysteresis nature, the ME effect the composites may find applications in memory devices such as ME data storage and switching. In the present work, the possibility of forming ferroelectric-ferromagnetic ceramics has been investigated. The Dielectric constants as a function of frequency were studied for different compositions. The loss tangent as a function of frequency was studied for different compositions. The combined ferroelectric and magnetic ordering will result in magneto-electric coupling in this material; further investigations are necessary which may throw light on improvement in the product properties.
\end{abstract}

Keywords: XRD, SEM, Dielectric constant, loss tangent

\section{Introduction}

A ME composite is a combination of ferrite and ferroelectric materials as their constituent phases. The ME effect arises due to the product property of the composite first introduced by "Van Suchetelene" [1] using $\mathrm{BaTiO}_{3}-\mathrm{CoFe}_{2} \mathrm{O}_{4}$ as the constituents. Magnetoelectric effect is a product property of two phases (ferrite and ferroelectric) in which the material becomes magnetized when placed in an electric field and electrically polarized when placed in a magnetic field. Due to ability of magnetoelectric conversion these ME composites are used in electronic devices as transducer, sensors, attenuator, band pass filter, phase shifters, broad band /wide band sensor, microwave application and ME memory applications[2- 9] . The purpose of this study is to realize the increase in resistivity of composites for gettings high ME voltage coefficient.

\section{Materials and Methods}

The components of the present ME composites namely ferrite, ferroelectric and their composites were prepared by ceramic method. Ferrite phase was prepared by using carbonates of nickel, zinc, cobalt and iron oxide. These chemicals were taken and mixed in appropriate molar proportion and ground in agate mortar for 23 hours. These compositions were presintered at $900^{\circ} \mathrm{C}$ for 12 hours. The ferroelectric phase is also prepared by same procedure using barium carbonate and titanium oxide. Ferroelectric phase is presintered at $1000^{\circ} \mathrm{C}$ for 12 hours. The samples of ME composites were prepared by mixing ferrite and ferroelectric phase in the ratio of $10,20,30,40$ and $50 \%$ of ferrite phase 10:90, 20:80,30:70, 40:60 and 50:50 respectively. These composites were presented at $1100^{\circ} \mathrm{C}$ for 12 hours. The pellets of ME composite were having thickness $2-3 \mathrm{~mm}$ and diameter $10-15 \mathrm{~mm}$ prepared by using hydraulic press. The remaining powder and pellets were final sintered at $1200^{\circ} \mathrm{C}$ for 12 hours.

The XRD patterns were taken by using X-ray diffractometer-Philips Model PW -1710, Wavelength of $\mathrm{CuK} \alpha$ radiation $-1.5418 \AA$, The rate of scanning $2^{\circ} \mathrm{per} / \mathrm{min}$ over range $20^{\circ}$ to $100^{\circ}$ were maintained. SEM of all samples was taken by using SEM model - JEOL JSM 6360. Grain size was calculated by Cottrell's method using relations

$$
\begin{aligned}
P_{L} & =\left(\frac{n}{2 \pi \cdot r}\right) M \\
L & =\frac{1}{P_{L}-1}
\end{aligned}
$$

Where, $\mathrm{P}_{\mathrm{L}}$ - Number of intercept of grain boundaries per unit length,

$\mathrm{M}$ - Magnification,

$\mathrm{r}$ - Radius of circle,

$\mathrm{n}$ - Number of intercepts in the circle and

L - Grain diameter 
The dielectric constant and loss tangent of the samples were studied as a function of frequency by using L.C.R meter bridge - Model HP $4284 \mathrm{~A}$ in the frequency range $100 \mathrm{~Hz}$ to $1 \mathrm{MHz}$.

\section{Results And Discussion}

XRD patterns of $\mathrm{Ni}_{0.5} \mathrm{Zn}_{0.3} \mathrm{Co}_{0.2} \mathrm{Fe}_{2} \mathrm{O}_{4}$ ferrite, $\mathrm{BaTiO}_{3}$ ferroelectric and $\mathrm{y}\left(\mathrm{Ni}_{0.5} \mathrm{Zn}_{0.3} \mathrm{Co}_{0.2} \mathrm{Fe}_{2} \mathrm{O}_{4}\right)+(1-\mathrm{y})$ $\left(\mathrm{BaTiO}_{3}\right) \mathrm{y}=0.1,0.2,0.3,0.4$ and 0.5 composite samples are show in fig. 1-3. The patterns show two sets of well defined peaks and with no any additional peaks apart from those of ferrite and ferroelectric phases. The ferrite phase has a cubic spinel structure with lattice parameter $a=8.36 \AA$. The ferroelectric phase has a tetragonal pervoskite structure with lattice parameters $\mathrm{a}=3.99 \AA$ and $\mathrm{c}=4.01 \AA$. The lattice parameters match fairly well with the lattice parameters of the components when present as single phases. It is also clear that, no chemical reaction between ferrite and ferroelectric phases has taken place, which is the primary requirement for observance of the ME effect of a composite. The lattice parameters, porosity, average grain size and dielectric constant of ferrite, ferroelectric and their composites are reported in table1.

Fig. 4 shows SEM micrographs of y $\left(\mathrm{Ni}_{0.5} \mathrm{Zn}_{0.3} \mathrm{Co}_{0.2} \mathrm{Fe}_{2} \mathrm{O}_{4}\right)+(1-\mathrm{y})\left(\mathrm{BaTiO}_{3}\right) \mathrm{y}=0.1,0.2,0.3,0.4$ and 0.5 composite samples. The porosity of the samples lies in the range $16 \%$ to $20 \%$. The porosity in composites decreases with the content of ferrite phase. The maximum porosity was observed for composite with $10 \%$ $\mathrm{Ni}_{0.5} \mathrm{Zn}_{0.3} \mathrm{Co}_{0.2} \mathrm{Fe}_{2} \mathrm{O}_{4}+90 \% \mathrm{BaTiO}_{3}$. It is also observed that with increase in ferrite content the average grain size increases.

Fig. 5 shows the frequency dependence of the dielectric constant $\left(\varepsilon^{\prime}\right)$ at room temperature for the studied samples. It can be seen from the figure that the value of dielectric constant decreases continuously with increasing frequency. The dielectric dispersion can be explained on the basis of Koop's two layer model and Maxwell-Wagner polarization theory [10-12]. Since an assembly of space charge carriers in the inhomogeneous dielectric structure described requires finite time to line up their axes parallel to an alternating electric field, the dielectric constant naturally decreases, if the frequency of the reversal field increases [13]. The decrease in the values of both $\varepsilon^{\prime}$ as the frequency increases can be related to the electron exchange between the $\mathrm{Fe}^{2+}$ and $\mathrm{Fe}^{3+}$ ions which can not follow the alternation of the electric field beyond a certain critical frequency [14]. The value of dielectric constant is found to be enhanced with increase in the content of ferrite in the composite. This high value can be explained on the basis of the fact that it has maximum number of ferrous ions whose exchange $\mathrm{Fe}^{2+} \leftrightarrow \mathrm{Fe}^{3+}$ given rise to maximum dielectric polarization. This is attributed to the different mechanisms of polarization that are expected to lag behind the field variations as the frequency increases. The high value of dielectric constant observed at lower frequencies is attributed to space charge polarization due to inhomogeneous dielectric structure. When in composites, ferroelectric regions are surrounded by nonferroelectric regions, they behave as relaxor ferroelectrics. Hence the dielectric constant is high. Dielectric constant remains independent of frequency at higher frequencies because of inability of electric dipoles to follow the alternating applied electric field.

Fig. 6 shows the variation of loss tangent $(\tan \delta)$ with frequency temperature for $y=0.1,0.2,0.3,0.4$ and 0.5 respectively. All the samples exhibit dielectric dispersion at lower frequencies. The plots are similar in behavior as that of dielectric constant with frequency.

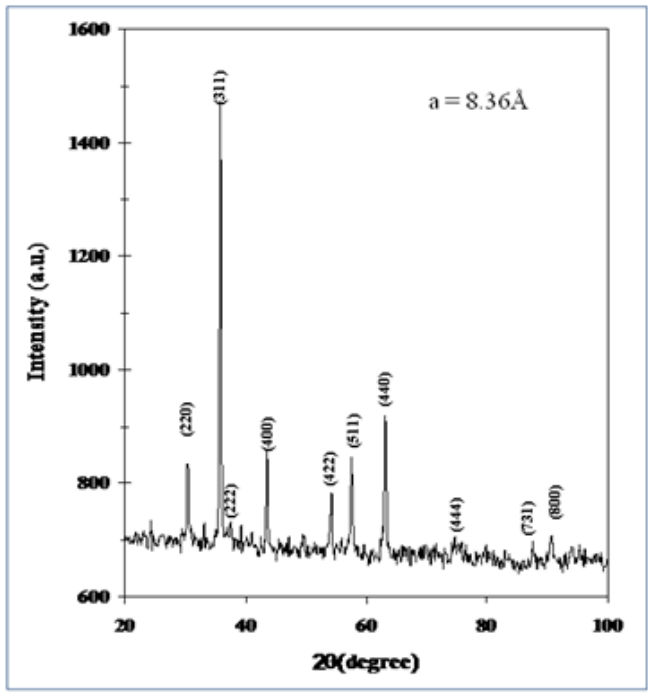

Fig. 1:- XRD pattern of $\mathrm{Ni}_{0.5} \mathrm{Zn}_{0.3} \mathrm{Co}_{0.2} \mathrm{Fe}_{2} \mathrm{O}_{4}$ ferrite

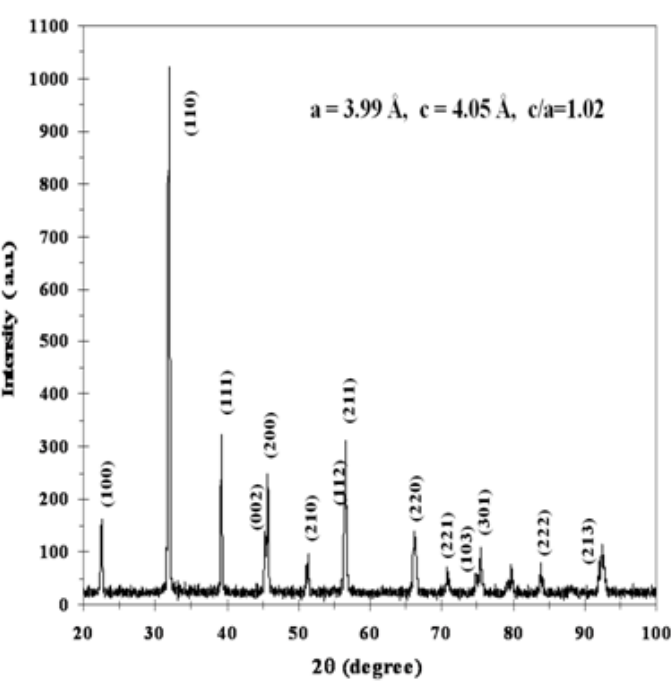

Fig 2:- XRD pattern of $\mathrm{BaTiO}_{3}$ ferroelectric 


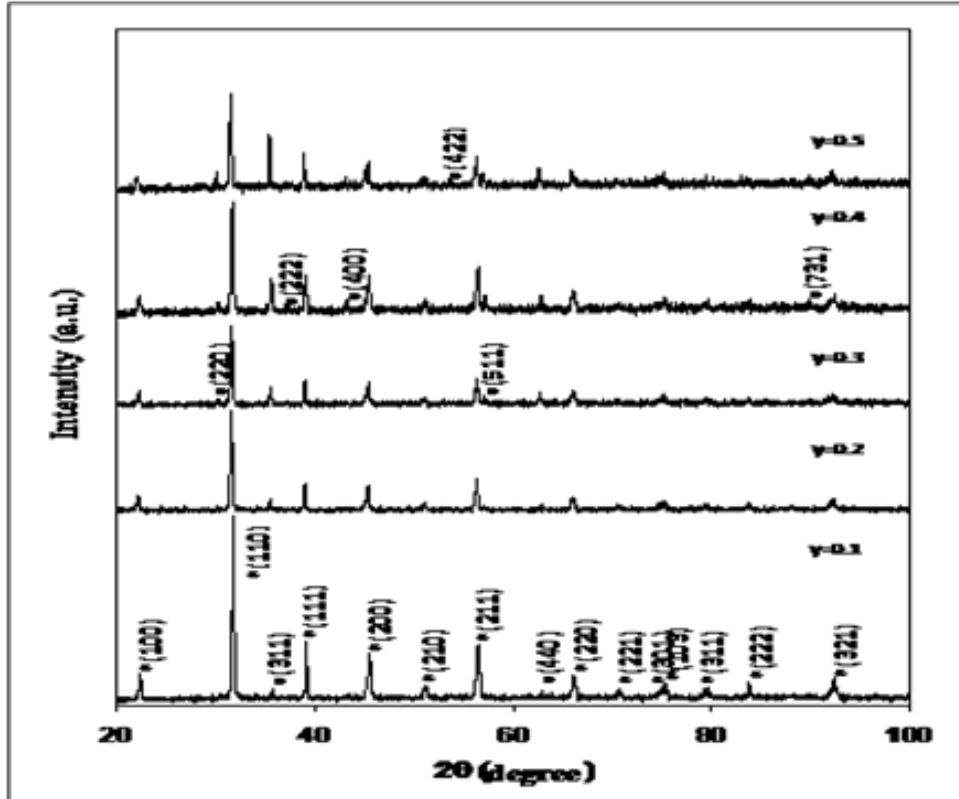

Fig 3:_XRD Patterns of y $\left(\mathrm{Ni}_{5} \mathrm{Zn}_{3} \mathrm{Co}_{2} \mathrm{Fe}_{2} \mathrm{O}_{4}\right)+(\mathrm{l}-\mathrm{y}) \mathrm{BaTiO}_{3}$ composites with

(a) $y=0.1$ (b) $y=0.2$, (c) $y=0.3$, (d) $y=0.4$ and (e) $y=0.5$
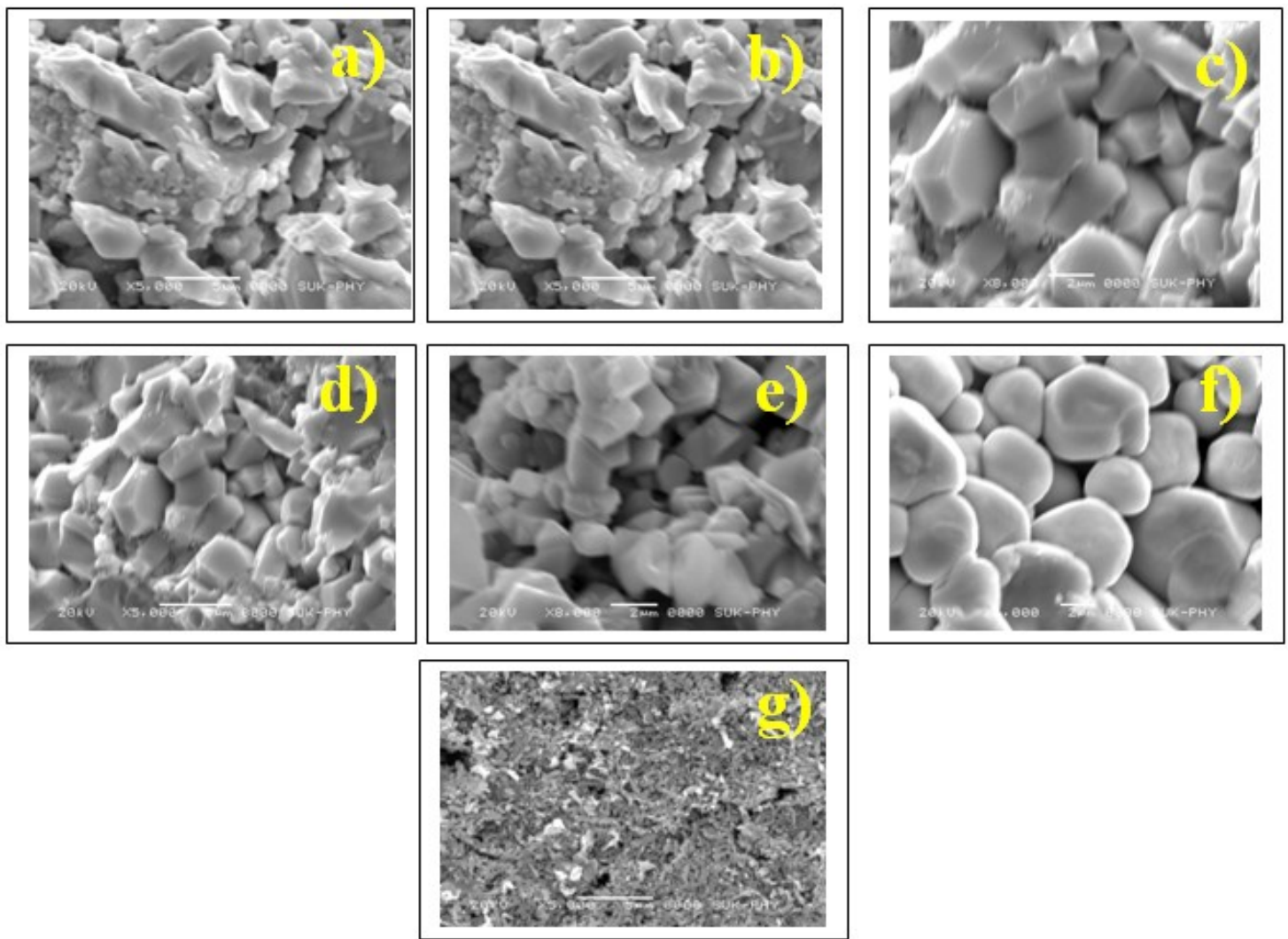

Fig. 4. SEM micrographs of $\mathrm{y}\left(\mathrm{Ni}_{0.5} \mathrm{Zn}_{0.3} \mathrm{Co}_{0.2} \mathrm{Fe}_{2} \mathrm{O}_{4}+(1-\mathrm{y})\left(\mathrm{BaTiO}_{3}\right) \mathrm{ME}\right.$ Composites where a) $\left.\mathrm{y}=0.1, \mathrm{~b}\right)$ $y=0.2, c) y=0.3, d) y=0.4, e) y=0.5$, f) $y=0$ and g) $y=1$ 

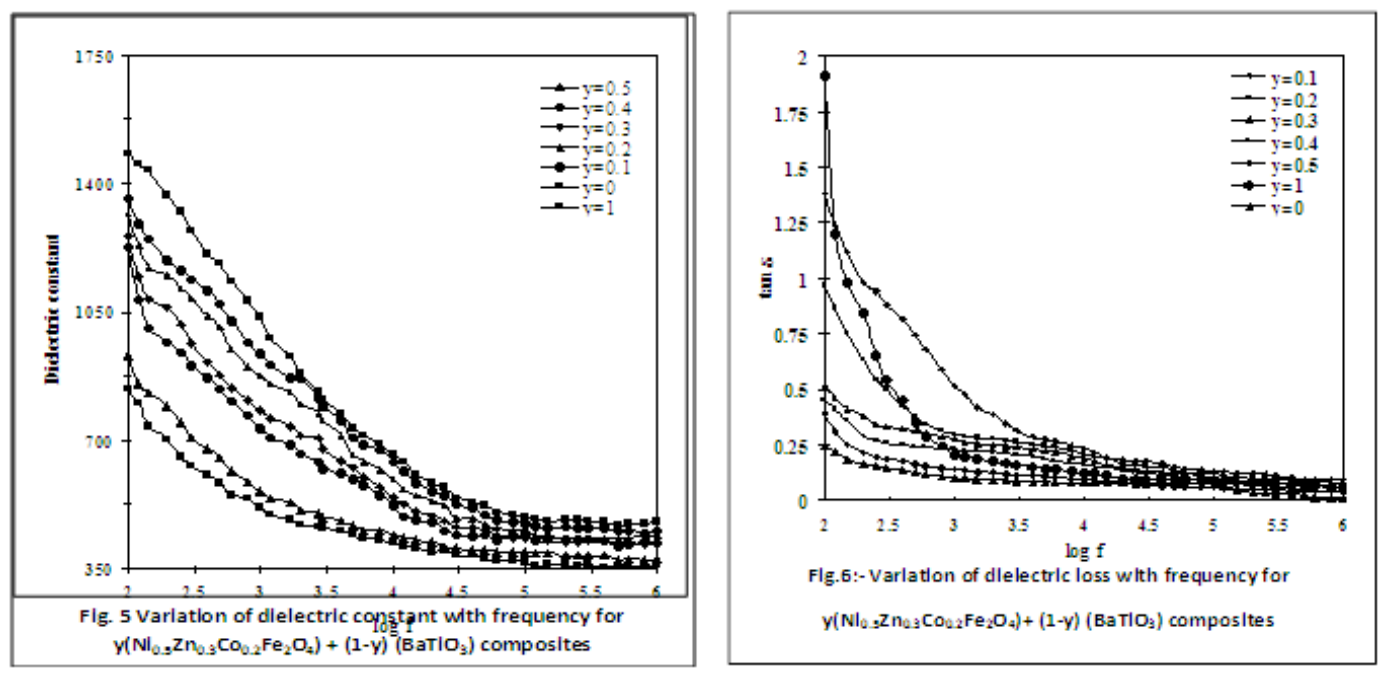

\section{Conclusions}

All the composites show the presence of ferrite and ferroelectric phases with corresponding maximum intensity peaks of (311) and (110) respectively. No extra lines were observed confirming the formation of composites without any impurity phases. The intensity of ferrite peak in the composites varies with its molar \% in the composites. The porosity of the samples lies in the range $16 \%$ to $20 \%$ which decides the resistivity of composites. The porosity in composites decreases with the content of ferrite phase. The maximum porosity was observed for composite with $10 \% \mathrm{Ni}_{0.5} \mathrm{Zn}_{0.3} \mathrm{Co}_{0.2} \mathrm{Fe}_{2} \mathrm{O}_{4}+90 \% \mathrm{BaTiO}_{3}$. Porosity and average grain size are inversely proportional to each other. The average grain size increases with increase in ferrite content. All the samples exhibit dielectric dispersion at lower frequencies. The dielectric constant is maximum for Barium Titanate and goes on increasing with ferrite content in composites. That may originate from the incorporation of ferrite phase in ferroelectric phase. The decrease in dielectric constant and loss tangent with frequency show dielectric dispersion in the lower frequency region.

[1]. J.V. Suchtelene. Philips Res. Repts. 27 (1972) 28

\section{Reference}

[2]. K.Uchino "Comprehensive Composite Materials" Elsevier, Amsterdam, Vol.5 (2000)

[3]. Leixiang Bian, Yumei Wen, Ping Li, Qiuling Gao, Min Zheng, Sensors and Actuators A 150 (2009) $207-211$.

[4]. Yanmin Jia , A'Xi Xue, Zhihua Zhou, Zheng Wub, Jianrong Chen ,Ke Ma, Yihe Zhang, Jiayi Zhou, Yu Wangd, Helen Lai Wa Chan, in t e r $\mathrm{n}$ a t i o $\mathrm{n}$ a 1 journal of hydrogen energy 38 ( 2013 ) $14915-14919$.

[5]. Jin Yang, Yumei Wen, Ping Li, Xianzhi Dai, Sensors and Actuators A 168 (2011) 358-364.

[6]. A.S. Tatarenko, M.I. Bichurin, Advances in Condensed Matter Physics, Article ID 28656210 (2012)

[7]. Chee-Sung Park, Shashank Priya, Advances in Condensed Matter Physics, Article ID 323165, 12, (2012).

[8]. Jian-Ping Zhou, Yang-Yang Guo, Zengzhe Xi, Peng Liu, Shuyu Lin, Gang Liu, Huai-WuZhang, Appl. Phys. Lett. 93 (2008) 152501.

[9]. C.M.Callewaert, J.V..Mantese, A.L.Micheli, D.A.Messuri, W.T. Phillips and S.A. Musiek. Comp. Engg. XVII, (2000) 50.

[10]. C.G.Koops, Phys.Rev., 83(1951)121.

[11]. J.C. Maxwell, "Electricity and magnetism" Oxford University press, London (1973)

[12]. K.W.Wagner. Ann. Physik., 40(1913)817.

[13]. R.P.Mahajan, K.K.Patankar, M.B.Kothale and S.A.Patil , Bull.Mater.Sci.,Vol.23, No.4, Aug 2000, pp.273-279.

[14]. V.R.K.Murty, J.Sobhanadari, Phys.Stst.Sol.A36, K133(1976). 